\title{
Essential considerations for the cementation of facets and ceramic laminates
}

\author{
Diogo de Azevedo Miranda* \\ Adjunct Professor, Department of Dentistry, Faculty of Medical Sciences and Health, Juiz De Fora, Brazil
}

\begin{abstract}
The facets and ceramic laminates are urgent for aesthetic recovery of the smile. When compared to direct directions with composite composition, they may be safer and predictable, provided the body is selected and selected, have a mastery of the materials and techniques involved. A career adhesive, the use in type of transmission, is a phase of critical, critical years, which should follow to follow the extortion of adherence and aesthetic species. Thus, after preparation, it is essential that the factor control be based on an adequate diet, with the aim of improving its capacity, with a minimum of aesthetic commitment. The use of resinous resources should be made as aesthetic and flexible particles. Thus, cementation-related parameters are important in determining the success and longevity of laminates, but should also be performed for case selection, adequate tooth preparation, and periodic maintenance.
\end{abstract}

\section{Introduction}

Aesthetics and the quest for ultraconservative treatments are increasingly on the rise. In a social context, where the vast majority of patients are focused on the beauty of magazine covers, today, disharmonious smiles are practically unacceptable.

More topics related to dental surgeons are those that improve concerns and damages, and among the various alternatives that today are able to meet this expectation, are like facets and ceramic laminates, which are among the most sought after. According to Park, et al. (2010: 77) [1] "Since its introduction in 1930 by Pincus, facets and ceramic laminates have made it the most popular and aesthetic treatment of anterior teeth".

Because they are conservative restorations and desired aesthetic results, their demand has considerably increased. Consumption of a type of immediate treatment and high acceptance, not being respected as techniques of preparation, conditioning and cementation, all work may be doomed to failure.

For Korkut, et al. [2] "to restore the aesthetics of a patient who is one of the main themes of dentistry, besides returning a function, form of color and phonation. The physical and positional abnormalities of previous medications may lead to treatment of patients with severe symptoms. On the other hand, as main indications of the ceramic facets are for the elements with the functions of color, shape, size, position, waves in the vestibular faces, resu - reations and for diastema closure. Another issue would be for minor corrections in occlusal relationships [3].

One of the major aesthetic evidences in restorations in anterior teeth was the development of a technique in which a greater interaction between the dental surface and the indirect restoration, all this maintaining the aesthetic factor. With this in mind, adhesive techniques have been developed to preserve the maximum dental structure and also to meet the aesthetic and restorative requirements of the patient [4].

\section{Planning and preparing techniques}

Nowadays, advancement in materials, improvement in manufacturing techniques, offer different alternatives for dentists to improve success rates in this type of treatment. Ceramic veneers not only restore the smile, but also allow the biomimetic recovery of the dental crowns. In cases requiring correction or change in tooth shape or position, changes in morphology, ceramic veneers exhibit promising aesthetic results when clinical procedures are carefully performed [5-9].

It is of extreme importance that the professional has the predictability of the treatment, so it is fundamental a good planning of the case so that possible flaws are minimized [3]. Ceramic veneers and composite resin restorations are predictable treatments, but present a difference in light reflection on their surfaces. In the aesthetic zone, as is the case of facets, this fact may represent a limiting factor for the choice of the restorative material, since the two substances interact differently in the light [6].

Recognizing the contraindications, advantages and disadvantages of this technique are also part of good planning of the case. They are contraindicated when the preparation does not allow the preservation of at least $50 \%$ of the enamel and when the margins are not totally located inside the enamel. Also, in cases where the patient presents inadequate occlusion, overbite, bruxism and other parafunctional habits [4,7]. Among the advantages of laminates is its good aesthetics and acceptance, not adversely affecting the periodontium, and also its minimally invasive preparation. Many facet and laminate cases can be done without local anesthesia. Among the disadvantages are the

*Correspondence to: Diogo de Azevedo Miranda, Rua Sampaio 330/602, Granbery, Juiz de Fora, Minas Gerais 36010-360, Brazil, E-mail: diogoodonto@yahoo.com.br

Key words: ceramic facets, laminates, cement, aesthetics

Received: December 31, 2018; Accepted: January 14, 2019; Published: January 16,2019 
difficulty in preparing the tooth, time until the preparation of the piece and also its fragility before being cemented, as any carelessness can cause cracks and / or fractures [7].

In the ceramic laminates, due to its thin thickness ( 0.3 to $0.9 \mathrm{~mm})$, the dental substrate can interfere in the final aesthetic result, so the correct choice of the ceramic system should involve an evaluation of the substrate, in the same way its thickness of the ceramic material to be used. Initially it is recommended to try to clear the dental substrate to neutralize the influence of the dental substrate and / or make use of several colors of resin cements [8].

In order to minimize the error rates in face preparation, it is recommended to use a protocol to facilitate its preparation. Large error rates are reported at the time of tooth preparation, either in the undefined margins and also in the depth to obtain a suitable thickness [7].

According to Hopp 7 , "obtaining" silicone guides ", especially those that allow a lateral view of the tooth, facilitates the monitoring of the wear and can be executed directly in the mouth, in cases where the enamel thickness of the is not altered ".

The use of spherical diamond tips (1011/1012) in high rotation is recommended for making the cervical canal. The depth of the preparation will depend on factors such as: the degree of tooth darkening, its inclination and also the need to restore the lost enamel [7]. The preparation for the central chute should be done also in high rotation, but with the use of a frustoconical diamond tip with rounded end. Its depth will depend on the same factors that determine the cervical canal.

The depth of preparation for facets ranges from 0.3 to $0.9 \mathrm{~mm}$, having a mean of 0.4 and $0.7 \mathrm{~mm}$. The incisal cover and cervical ends in the form of a knife blade can better withstand occlusal forces [3]. The proximal contacts of natural teeth should be maintained whenever possible, as they are a very difficult anatomical feature to reproduce, they also prevent tooth movement before installing the laminate, as well as facilitating final adjustments to the facet, making it easier to adhesion and finishing, are effective in plaque control [7].

\section{Treatment of ceramics, substrate conditioning and ce- mentation}

For the success of any restorative preparation, in addition to a good cavitation preparation, the cementing techniques are of equal importance for longevity, aesthetic aspect, as well as good periodontal health. It is the step that requires greater care by the operator, either in the technical sequence or in the material used [9].

According to Anchieta, et al. [10] "The resin cement is the material of choice for bonding the ceramic to the dental substrate and can also dictate the final aesthetic appearance and strength of the restoration. These cements present several options of color and opacity, very important in the manufacture of ceramic laminates, because the color of the cement to be used may interfere in some way in the final aesthetic result, especially if the ceramic system used has some translucency [8].

Among the main causes of faults in veneers and ceramic laminates, due to the short durability or fractures, it is precisely at the moment of cementation that clinical success depends directly on a correct cementation of these indirect restorations [4]. Photoactivated resin cements are currently indicated for cementation of ceramic restorations. These restorations require an internal treatment according to their ceramic composition in order to improve cement-ceramic bonding. Resin cements bind to the substrate by friction and abrasion $[11,12]$.

The internal surface of the ceramic laminate is treated with the hydrofluoric acid conditioning on the inner surface of the laminate, creating microporosities and promoting a cleaning of the part and improving its adhesion to the dental substrate [3]. In order to improve the durability of the restoration, the literature indicates that the concentration of hydrofluoric acid is time-dependent: increasing the concentration of acid, decreasing the exposure time to the acid and vice versa $[3,4]$. Then remove the acid with air-water jets and dry the inner surface well, resulting in a rough, opaque region. The silanization is then carried out for 60 seconds. Another important fact is that the operator must know the composition of the ceramic in this stage. When choosing feldspathic ceramics, the conditioning time varies from 120 to 240 seconds. For ceramics based on lithium disilicate, this time varies from 20 to 40 seconds depending on the concentration of the acid [13].

In relation to the conditioning of the dental surface, it is indispensable to isolate the operative field, controlling the humidity and preventing the contamination of the dental substrate. It should be conditioned with $37 \%$ phosphoric acid, 30 seconds for the enamel and 15 seconds for the dentin, followed by washing and drying for the adhesive system to be applied [3].

Among all the steps taken, cementation is one of the most critical steps. For high success rate and durability, the cementation should be adhesive. The choice of cementitious agent should be made with extreme care, the wrong choice may cause a change in the coloring of the laminated edges. To facilitate color choice, manufacturers offer cement tubes for color testing, called cements testing or "try-in" [3].

As for the type of adhesive, the dentist should pay attention to the conventional or self-etching system. One merit to be raised is that the universal adhesives tend to be more yellowish and this may alter the color of the cement previously. Therefore, there are already stickers with good properties and, practically transparent, not to cause this type of problem [11].

In order to perform a good cementation, the cement must be homogeneously applied throughout the surface of the ceramic laminate, thus avoiding the lack of cement in some region of the preparation, remembering that the thickness of the cement has a great influence on the distribution of the stresses, thereby it is possible to minimize adhesive and color glitches [3]. The traditional cementation where the dentin adhesive is applied and polymerized with the laminate in position is related to adhesion failures. At no time of the step the application of the adhesive system must be followed by photoactivation, as this would create a "pre-polymerized film" and would prevent perfect adaptation of the part in the substrate $[4,11]$.

\section{Longevity}

The ceramic is highlighted as a restorative material that fills the aesthetic, biological, mechanical and functional requirements. Their growing demand within dentistry has provided an optimization in their properties, making them thus materials with undeniable advantages in terms of color stability, stain resistance and wear [12].

In addition to the aesthetics in the treatments in anterior teeth, the patient is also demanding that it be a durable treatment, one of the determining factors for a good durability is the precision in the marginal settlement of these restorations, it becomes fundamental 
for the maintenance of good health of the dental organ as well as the surrounding tissues [13].

One important physical property for the longevity of a restoration is fracture resistance. It is a measure of the ability of a material to resist the growth of a crack, i.e., a measure of the amount of energy required to cause the crack. Clinically, restorations are not loaded for failure as the flexural strength test is done, instead, millions of less critical loads (mastication) are applied to them. Materials eventually fail because of this cyclic fatigue by the propagation of cracks. Thus, materials with higher fracture resistance are more clinically ideal as more energy is needed to cause crack growth. Other factors, such as stress corrosion (chemical-assisted crack growth) and residual material failure significantly affect the final strength of a material in its final state [14].

For Román-Rodríguez, et al. [11] "with a good cement-ceramic bond increases the strength of the restoration, as well as decreasing the possibility of dental fractures, as well as preventing secondary caries and color change of the cement and decreasing the likelihood of detachment in cases of short or tapered preparations".

Cements that present amine as an activator, such as chemically activated cements and duals, can cause changes in the color of the restoration over time, compromising its longevity and esthetics and therefore should be discarded as a possibility for cementation. Failures in techniques such as moisture contamination or insufficient photoactivation can also compromise color stability over time [8].

According to Gonzalez, et al. [3] "another factor that may interfere in the indirect restoration longevity is the resin dentin interface, being on a surface with $80 \%$ or more of the dentin surface". The preparation, previously discussed, being made entirely in enamel is a factor that helps in the success of adhesion.

\section{References}

1. Park DJ, Yang JH, Lee JB, Kim SH, Han JS (2010) Esthetic improvement in the patient with one missing maxillary central incisor restored with porcelain laminate veneers. $J$ Adv Prosthodont 2: 77-80. [Crossref]

2. Korkut B, Yanikoglu F, Günday M (2013) Direct composite laminate veneers: Three case reports. J Dent Res Dent Clin Dent Prospect 7: 105-111.

3. Gonzalez MR (2011) Falhas em restaurações com facetas laminadas: uma revisão de literatura de 20 anos. Rev Bras Odontol Rio de Janeiro/RJ 68: 238-243.

4. Pini NP, Aguiar FH, Lima DA, Lovadino JR, Terada RS, et al. (2012) Advances in dental veneers: materials, applications, and techniques. Clin Cosmet Investig Dent 4: 9-16. [Crossref]

5. Türkaslan S, Ulusoy KU (2009) Esthetic rehabilitation of crowed maxillary anterior teeth utilizing ceramic veneers: a case report. Cases Journal 2: 1-5.

6. Narcisi EM, DiPerna JA (1999) Multidisciplinary full-mouth restoration with porcelain veneers and laboratory-fabricated resin inlays. Pract Periodontics Aesthet Dent 11: 721-728. [Crossref]

7. Hopp CD, Land MF (2013) Considerations for ceramic inlays in posterior teeth: A review. Dovepress journal: Clinical, Cosmetic and Investigational Dentistry 5: 21-32.

8. Cardoso PC (2001) Importância da Pasta de Prova (Try-In) na Cimentação de Facetas Cerâmicas - Relato de Caso. Rev Odontol Bras Central 20: 166-171.

9. Altintas SH, Tak O, Secilmis A, Usumez A (2011) Effect of provisional cements on shear bond strength of porcelain laminate veneers. Eur J Dent 5: 373-379. [Crossref]

10. Anchieta RB1, Rocha EP, de Almeida EO, Junior AC, Martini AP (2011) Bonding allceramic restorations with two resins cement techniques: A clinical report of three-year follow-up. Eur J Dent 5: 478-485. [Crossref]

11. Román-Rodrígues JL (2013) Bond strength of selected composite resin- cements to zirconium-oxide ceramic. Med Oral Patol Oral Cir Bucal 18: 115-123.

12. Carvalho RLA (2012) Indicações, adaptação marginal e longevidade clínica de sistemas cerâmicos livres de metal: uma revisão de literatura. Int J Dent 11: 55-65.

13. Gordilho AC (2009) A adaptação marginal dos principais sistemas de cerâmica pura. Revista Odonto 17: 82-92.

14. Shenoy A, Shenoy N (2010) Dental ceramics: An update. Journal of Conservative Dentistry 13: 195-203.

Copyright: (C2019 de Azevedo Miranda D. This is an open-access article distributed under the terms of the Creative Commons Attribution License, which permits unrestricted use, distribution, and reproduction in any medium, provided the original author and source are credited. 